\title{
SYNTHESIS OF PERIODIC MESOPOROUS SILICA THIN FILMS
}

$$
\text { SAN096-12976 }
$$

M.T. ANDERSON, J.E. MARTIN, J.G. ODINEK, P. NEWCOMER

$$
\text { CONF. } 960401--44
$$

Sandia National Laboratories, P.O. Box 5800, Albuquerque, NM 87185

\section{ABSTRACT}

We have synthesized periodic mesoporous silica thin films (PMSTF) from homogeneous solutions. To synthesize the films a thin layer of a $\mathrm{pH}=7$ micellar coating solution that contains TMOS is dip- or spin-coated onto silicon wafers, borosilicate glass, or quartz substrates. Ammonia gas is diffused into the solution and causes rapid hydrolysis and condensation of the TMOS and the formation of periodic mesoporous thin films within $\sim 10$ seconds. The combination of homogeneous solutions and rapid product formation maximizes the concentration of desired product and provides a controlled, predictable microstructure. The films have been made continuous and crack-free by optimizing initial silica concentration and film thickness.

\section{INTRODUCTION}

Periodic mesoporous metal oxides can be formed by templating a molecular source of metal oxide around surfactant arrays [1-5]. Thin films of hexagonal periodic mesoporous silica have been previously synthesized from inhomogeneous solutions [6-8] or by pressing premade powders onto substrates [9]. We sought to make solutions from which periodic mesoporous silica thin films (PMSTF) could be dip- or spin-coated. There are three challenges: (1) to make homogeneous solutions in order to avoid incorporating byproducts in the film (e.g. amorphous silica, excess surfactant), (2) to rapidly form the products in order to avoid incorporation of unreacted species from the solution in the film (e.g. partially hydrolyzed alkoxides), (3) to control the microstructure of the film to avoid large variations in particle size and agglomeration as seen in bulk preparations [1-5].

To meet all of these challenges, we rapidly form films that have a controlled, predictable microstructure from homogeneous solutions. We accomplish this by using a readily hydrolyzable source of silica (tetramethoxysilane, TMOS) and a homogenizing agent to ensure that all of the reagents are dissolved prior to film formation. We can make continuous thin films of aggregated 20-500 $\mathrm{nm}$ periodic mesoporous particles; within each particle exists the familiar hexagonal array of 2-4 nm diameter channels. The film thickness can be varied from a few hundred nanometers to several microns. The films are stable to calcination at $500{ }^{\circ} \mathrm{C}$ and exhibit extremely high surface areas. The films are being evaluated as high surface area, size-selective coatings for surface acoustic wave (SAW) sensors.

\section{EXPERIMENTAL}

A typical synthesis of periodic mesoporous silica involves mixing deionized water, methanol, cetyltrimethylammonium bromide $\left(\mathrm{C}_{16} \mathrm{TMABr}\right), \mathrm{NaOH}$ or $\mathrm{NH}_{3}$ gas, and tetramethoxysilane (TMOS). The water to methanol ratio is also fixed at $75: 25 \mathrm{w} / \mathrm{w}$ and the micellar solutions, before the addition of TMOS, are 2, 4, 8, $16 \mathrm{wt} \%$ in $\mathrm{C}_{16} \mathrm{TMABr}$ solutions. After addition of the TMOS, the silica concentrations are $0.4,0.76,1.38,2.33 \mathrm{M}$, respectively. The $\mathrm{H}_{2} \mathrm{O}$ : $\mathrm{Si}$ ratios $(h)$ vary from 97 to 12 in this range.

X-ray diffraction data were collected on crushed monoliths using a Scintag PAD V instrument equipped with nickel-filtered $\mathrm{Cu} \mathrm{K}_{\alpha}$ radiation. High resolution magic angle spinning 


\section{DECCLAMER}

Portions of this document may be illegible in electronic image products. Images are produced from the best available original document. 


\section{Disclaimer}

This report was prepared as an account of work sponsored by an agency of the United States Government. Neither the United States Government nor any agency thereof, nor any of their employees, makes any warranty, express or implied, or assumes any legal liability or responsibility for the accuracy, completeness, or usefulness of any information, apparatus, product, or process disclosed, or represents that its use would not infringe privately owned rights. Reference herein to any specific commercial product, process, or service by trade name, trademark, manufacturer, or otherwise does not necessarily constitute or imply its endorsement, recommendation, or favoring by the United States Government or any agency thereof. The views and opinions of authors expressed herein do not necessarily state or reflect those of the United States Government or any agency thereof.

This report has been reproduced directly from the best available copy.

Available to DOE and DOE contractors from the Office of Scientific and Technical Information, 175 Oak Ridge Turnpike, Oak Ridge, TN 37831; prices available at (615) 576-8401.

Available to the public from the National Technical Information Service, U.S. Department of Commerce, 5285 Port Royal Road, Springfield, VA 22161; phone orders accepted at (703) 487-4650. 


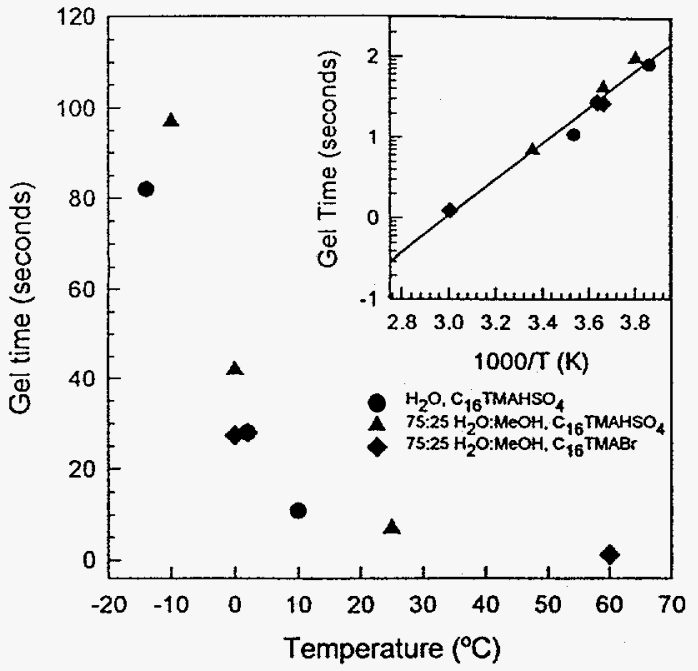

Figure 1. The gel time exhibits Arrhenius behavior over a large temperature range and is notably independent of solvent type or surfactant counterion, which indicates hydrolysis of TMOS is the rate-limiting step.
${ }^{29} \mathrm{Si}$ NMR spectra were recorded on powder samples using a Chemagnetics instrument equipped with a 4.7 Tesla magnet. A JOEL $1200 \mathrm{EX}$ transmission electron microscope (TEM) with ASID (SEM) attachment was used to observe the microstructure and grain size of the powder samples on holey carbon film of a $3 \mathrm{~mm}$ copper grids. Static and dynamic light scattering measurements were made with a $63 \mathrm{~mW}$ NEC He-Ne laser using a 256 channel Langley-Ford correlator. A Malvern index-matching temperaturecontrolled scattering vat, a Malvern detector assembly, using an RCA FW130 photomultiplier tube, and an Aerotech 12 inch steppermotor-drive goniometer complete the basic light scattering hardware.

\section{RESULTS}

\section{$\underline{\text { Solution Homogeneity and Reaction Kinetics }}$}

The first of our goals is to make homogeneous solutions from which to dip- or spin-coat films. In order to make homogeneous solutions the reagents should be molecular (i.e. alkoxides) and must all be dissolved prior to product formation. The surfactant and base are dissolved in solution prior to the addition of the silica source, so the key then is to solubilize the alkoxide prior to the formation of periodic mesoporous silica. Silicon alkoxides are not initially soluble in a pure water, but it is well known that alcohols act as homogenizing agents for alkoxides in aqueous solutions, so we added methanol to the precursor solution. As reported elsewhere [10], adding $25 \mathrm{wt} \%$ methanol actually improves the long-range order and yield of bulk products.

The second goal is to speed the reaction kinetics. This is essential because when TEOS or non-molecular sources of silica are used a wide variety of species are simultaneously present in the reaction mixture, such as, partially hydrolyzed alkoxides $\left(\mathrm{Si}\left(\mathrm{OCH}_{3}\right)_{4 \times \mathrm{x}}(\mathrm{OH})_{\mathrm{x}}\right.$, silicate oligomers, colloidal products, amorphous silica, free surfactant, and precipitated product. Obviously this is not an optimal mixture from which to make pure, continuous PMSTFs. Fortunately, the time for phase separation of the products is accelerated by $\sim 2$ orders of magnitude when TMOS is used as the silica source instead of tetraethoxysilane (TEOS). In fact, periodic mesoporous silica forms within 5-7 seconds at room temperature with TMOS, Figure 1, and aggregation and gelation occur in less than 1 second. This nearly instantaneous transformation from homogeneous solution to gelled product effectively eliminates the broad distribution of species found when TEOS is the silica source.

The third goal is to control the morphology and agglomeration of particles. Control of morphology is provided when TMOS is used as the silica source. The product forms aggregated colloidal particles that are $20-500 \mathrm{~nm}$ diameter. The particles form a continuous gel network in which the particle-particle contact area is minimal, which provides ready access to the intraparticle porosity for adsorbing gases.

As shown in Figure 1, the time to form a product is increased as the temperature is decreased. We reasoned that a homogeneous solution could be deposited onto a substrate and spun before it gelled. (It is possible to form homogeneous micellar solutions at temperatures as low as $-14{ }^{\circ} \mathrm{C}$ if $\mathrm{C}_{16} \mathrm{H}_{33} \mathrm{~N}\left(\mathrm{CH}_{3}\right)_{3} \mathrm{HSO}_{4}$ is used as the surfactant rather than $\mathrm{C}_{16} \mathrm{H}_{33} \mathrm{~N}\left(\mathrm{CH}_{3}\right)_{3} \mathrm{Br}$ ). The 


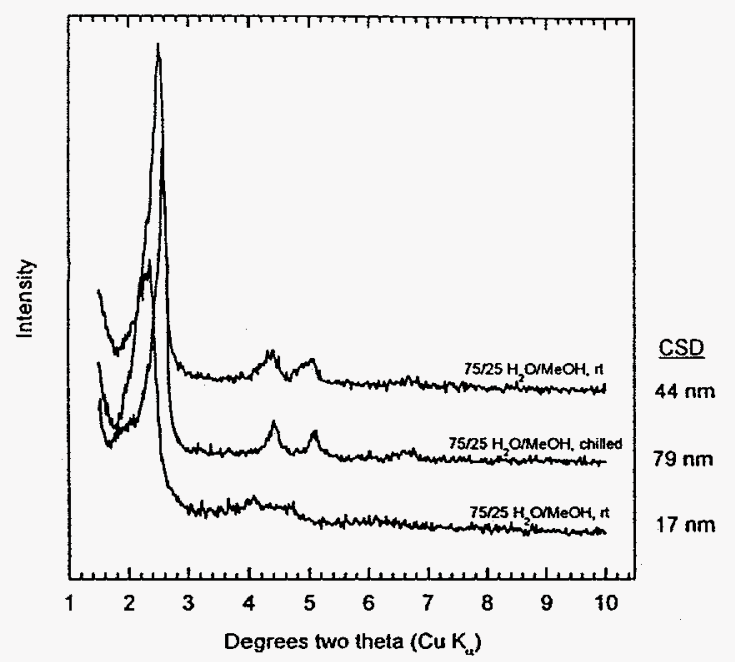

Figure 2. Diffraction data from bulk samples made with $\mathrm{NH}_{3}$ gas catalyst. The coherent scattering domain (CSD) sizes are shown. The CSD depend on solution temperature and agitation.

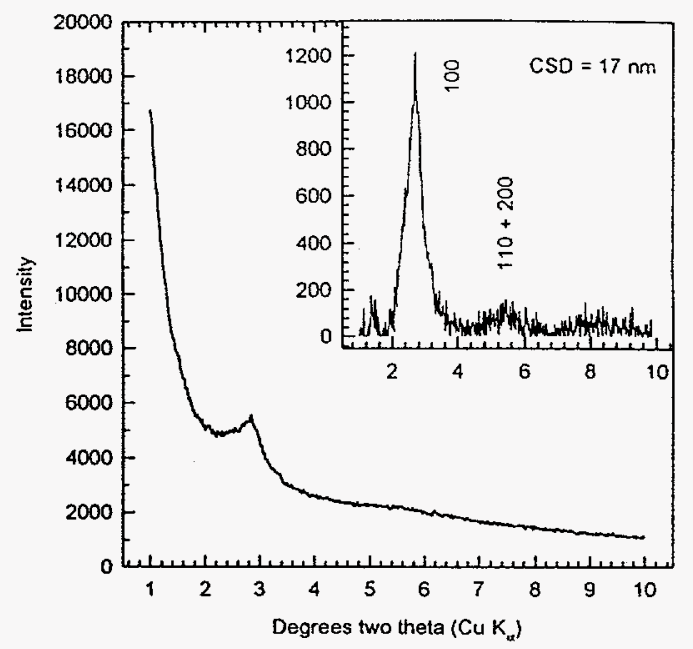

Figure 3. Diffraction data from a film made by gassing with $\mathrm{NH}_{3}$ a dip coated solution that contained $\mathrm{C}_{16} \mathrm{TMABr}, 75: 25$ water:methanol, and $1.38 \mathrm{M}$ silicon. The inset shows the data with the background subtracted.

shear forces during spinning provide a liquid layer of essentially constant thickness, except at the extreme periphery of the substrate. The speed at which the substrate is spun thus fixes the reaction volume and the final thickness of the film.

PMSTFs were prepared by chilling $75: 25$ water:methanol solutions to $0{ }^{\circ} \mathrm{C}$, squirting them on a room temperature substrate, and spinning at 500 to $9000 \mathrm{rpm}$. Films of PMS were formed, but with incomplete coverage. The key problems were predictably controlling the kinetics and deciding exactly when to begin spinning. If films were spun too early, convection cells, induced by preferential evaporation of the methanol, caused very uneven films to form. If they were spun too late, particles formed during spinning and were subject to the physics of the spinning substrate. In most cases films with incomplete coverage formed; in some cases unusual radial lines of silica formed that emanated from the center of the substrate, in other cases dendritic diffusion limited aggregates (DLA) of surfactant formed.

From these early studies we concluded that the rapid kinetics observed for the TMOS system are advantageous in that we avoid a wide distribution of species in solution, and, as we generally use aqueous-based solutions that have a high surface tension, we can form films before the solution dewets. Nonetheless, without strict temperature control and precise timing the kinetics are problematic in that the exact instant of product formation is difficult to predict accurately enough to get continuous films. Thus a method is needed to predictably delay the product formation until the coating solution is on the substrate in the appropriate thickness.

\section{The 4+1 Approach: A pH switch}

To delay the product formation we sought to form a stable coating solution, deposit it on a substrate, spin or drain it to the desired thickness, and rapidly form product. From studies on bulk samples, we find that the product does not form until after the last reagent is added. Normally the last reagent added is TMOS, but we surmised that a stable coating solution could be formed by making a $\mathrm{pH}=7$ water:methanol:surfactant:TMOS solution; the solution could then be deposited on the substrate, and then the catalyst could be added. In order to provide rapid and thorough infiltration of the catalyst into the liquid reaction layer, we added the catalyst as a gas, in this case ammonia. 


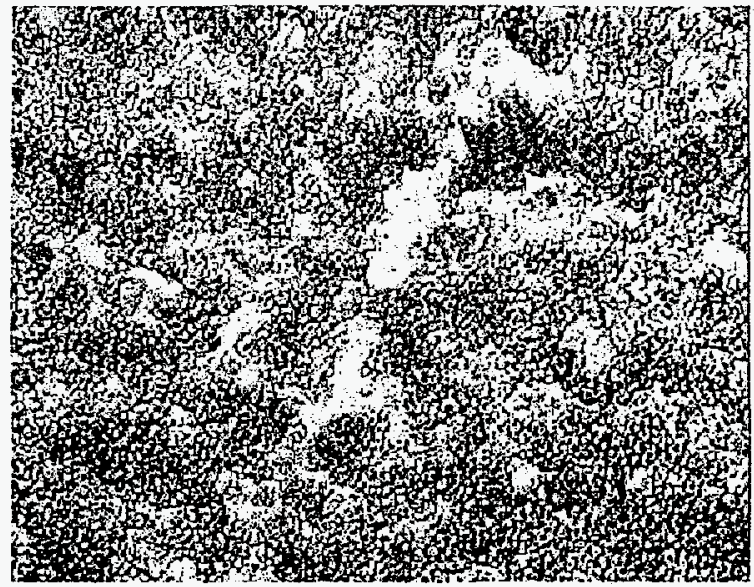

Figure 4. Optical micrograph (1250 x) of a dipcoated thin film made from a $2 \% \mathrm{C}_{16} \mathrm{TMABr}, 75: 25$ water:methanol, $0.4 \mathrm{M}$ TMOS solution gassed with $\mathrm{NH}_{3}$.

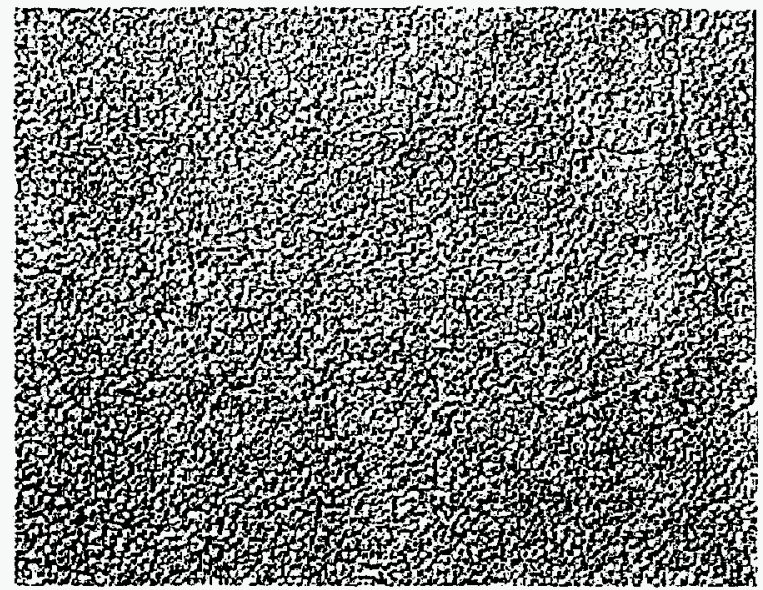

Figure 5. Optical micrograph $(1250 \mathrm{x})$ of a dipcoated thin film made from a $8 \% \mathrm{C}_{16} \mathrm{TMABr}, 75: 25$ water:methanol, $0.4 \mathrm{M}$ TMOS solution gassed with $\mathrm{NH}_{3}$.

To confirm that we could form periodic mesoporous silica by this approach, we made bulk products by exposing the neutral reaction solution to ammonia gas. X-ray diffraction clearly shows that this process leads to periodic mesoporous silica, Figure 2. Furthermore, the product forms in the top few millimeters of the static liquid within 10s. By dip coating the neutral solution onto silicon substrates and then gassing with ammonia, we were able to make films with diffraction patterns characteristic of PMS, Figure 3. Thus we now have a stable coating solution that can be spun or dipped onto substrates, and we have a $\mathrm{pH}$ "switch" that allows PMS to be formed in a few seconds.

The neutral coating solution remains clear for $\sim 90$ minutes before a transparent or translucent chemical gel forms. Thus we wanted to determine the optimal time at which to dip the solution. We examined the evolution of silicate and surfactant species with static light scattering, dynamic light scattering, and ${ }^{29} \mathrm{Si}$ NMR.

The NMR measurements show that for an $[\mathrm{Si}]=1.38 \mathrm{M}$ solution, $\mathrm{Q}^{0}{ }_{10 \mathrm{H}}$ form after $\sim 10$ minutes, $Q^{1}$ species form after $\sim 30$ minutes, $Q^{2}$ species begin to form after $\sim 60$ minutes, and $Q^{3}$ and $\mathrm{Q}^{4}$ species begin to form after $\sim 70$ minutes. From light scattering measurements we see that the species in solution - micelles, and partially hydrolyzed alkoxide monomers and oligomers do not grow significantly in size for about $1 \mathrm{~h}$ in a $1.38 \mathrm{M}$ silica solution. From the dynamic light scattering we see that the hydrodynamic radii of the primary species in solution (presumably micelles encrusted with charged silicate ions and bromide ions) are only about twice the size of the micelle radii water:methanol solution. The chemical gels that form at $\sim 90$ minutes are amorphous in X-ray diffraction and therefore are not a desired product. Thus we find the optimal time window to deposit the coating solution is between 10 and 60 minutes. In this range the silicate precursor have begun to hydrolyze so they wet more hydrophilic substrates, and the silicate species are still small so they do not frustrate the transformation to PMS.

\section{Optimizing Film Thickness, Coverage, and Uniformity}

The $4+1$ approach leads to periodic mesoporous silica films, but the film thickness, coverage, and uniformity has to be optimized. The ultimate thickness of the film is determined by the coating conditions, that is, spin speed or withdrawal rate of the substrate, along with the initial silica concentration. The coverage depends on the initial concentration of silica in the coating solution. Figures 4 and 5 show the difference in coverage for a film made from a [Si] $=$ 


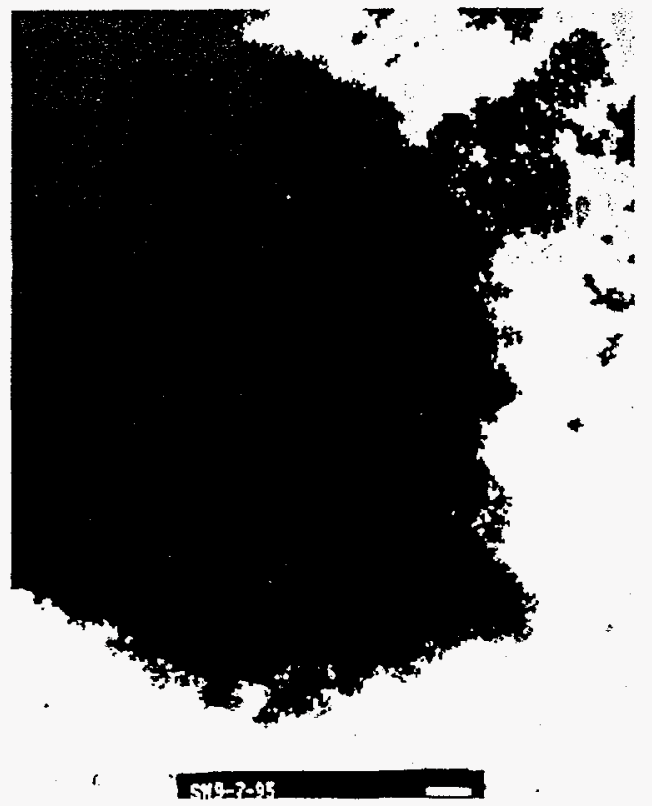

Figure 6. Low magnification (scale bar $=500 \mathrm{~nm}$ ) transmission electron micrograph of thick film made as in Figure 5. The film was scraped off the substrate so that it could be imaged in transmission.

$0.40 \mathrm{M}$ solution versus one made from $\mathrm{a}[\mathrm{Si}]=$ $1.38 \mathrm{M}$ solution. The coverage is continuous and complete for the latter, whereas it is not continuous for the former. In general we find that for continuous coverage it is best to use solutions that have an initial silica concentration of $\sim 1.3 \mathrm{M}$ or greater.

The uniformity of the films depends on the wetting characteristics of the solution and the differences in evaporation rate of the components of the liquid phase. The preferential evaporation of methanol in a water:methanol system leads to two rather severe problems: (These systems give films with the longest range order of the hexagonal pore system and are thus preferred). in thin films it rapidly concentrates the fluid in water, which causes the surface tension to increase and the solution to dewet, and it leads to macroscopic convective rolls in the liquid which can cause films to have nonuniform thicknesses and incomplete coverage. (These rolls can be imaged with an optical microscope by placing polystyrene latices in a water: methanol mixture.)

To reduce the difference in evaporation rates of the water and methanol, we built a simple controlled atmosphere chamber (CAC). The coating solution is in a vial within the chamber. The bottom of the chamber is filled with a water:methanol mixture so that the fugacity of the methanol in the liquid at the bottom of the chamber approximately matches that in the coating solution on the film. This leads to essentially no net evaporation of methanol from the coating solution, which slows the dewetting and leads to films with complete, continuous coverage and uniform thickness.

\section{Microstructure and Sorptive Properties}

Scanning electron microscopy and transmission electron microscopy, Figure 6, shows that the PMSTFs consist of aggregated submicron ellipsoidal or spherical particles. X-ray diffraction and transmission electron microscopy reveal that within each particle there is a periodic hexagonal array of 1-dimensional, unimodal $3 \mathrm{~nm}$ channels. The primary particle size tends to be $20-100 \mathrm{~nm}$ when ammonia gas is used as catalyst and $\sim 150-500 \mathrm{~nm}$ when sodium hydroxide is used as a catalyst.

PMSTF were coated on ST-cut surface acoustic wave (SAW) devices. The coated devices were calcined at $500^{\circ} \mathrm{C}$ in air to remove the organic template from the film. Films less than $1 \mu \mathrm{m}$ did not crack during the calcination procedure. Films greater than $\sim 1 \mu \mathrm{m}$ thick had large cracks after calcination and were not considered further. Nitrogen sorption measurements at $77 \mathrm{~K}$ were performed on a calcined film that are $<1 \mu \mathrm{m}$ thick using an apparatus that continuously varies the relative ratio of $\mathrm{N}_{2}$ and the nonadsorbing carrier gas $\mathrm{He}$ from $\mathrm{N}_{2}: \mathrm{He}=0.03$ to 1.0 [11]. From BET analysis of the region from $\mathrm{P} / \mathrm{P}_{0}=0.10$ to 0.18 in the adsorption branch, the surface area enhancement is $\sim 145 \mathrm{~cm}^{2} / \mathrm{cm}^{2}$. From this value we conservatively estimated the film surface area per gram is $\sim 300 \mathrm{~m}^{2} / \mathrm{g}$ based on a film thickness of $1 \mu \mathrm{m}$ and a bulk density of $\sim 0.5 \mathrm{~g} / \mathrm{cc}[12]$. 


\section{CONCLUSIONS}

We have developed and optimized the synthesis of periodic mesoporous thin films from homogeneous solution. The keys to continuous films with complete coverage are: controlling formation kinetics through the use of TMOS as the silica source, the addition of the catalyst in the form of ammonia gas, the use of a controlled atmosphere chamber to control evaporation of the coating solution, and the use of concentrated coating solutions. The films exhibit large surface areas. Films deposited on SAW devices maximize the device sensitivity and provide a size selective porous coating in which the pore diameter can be tuned from 15 to $50 \AA$.

\section{ACKNOWLEDGMENTS}

We acknowledge Roger Assink (SNL) and S. Prabakar for the NMR measurements. This work was funded by the United States Department of Energy under Contract Number DE-AC04-94AL8500.

\section{REFERENCES}

1. J. S. Beck, J. C. Vartuli, W. J. Roth, M. E. Leonowicz, C. T. Kresge, K. D. Schmitt, C. T-W. Chu, K. H. Olson, E. W. Sheppard, S. B. McCullen, J. B. Higgins, and J. L. Schlenker, J. Am. Chem. Soc. 114, pp. 10834-10843 (1992).

2. C. T. Kresge, M. E. Leonowicz, W. J. Roth, J. C. Vartuli, and J. S. Beck, Nature 359, pp. 710-712 (1992).

3. Q. Huo, D. I. Margolese, U. Ciesla, P. Feng, T. E. Gier, P. Sieger, R. Leon, P. M. Petroff, F. Schuth, and G. D. Stucky, Nature 24, pp. 317-321 (1994).

4. Q. Huo, D. I. Margolese, U. Ciesla, D. G. Demuth, P. Feng, T. E. Gier, P. Sieger, A. Firouzi, B. F. Chmelka, F. Schuth, and G. D. Stucky, Chem. Mater. 6, pp. 1176-1191 (1994).

5. C.-Y. Chen, H-X. Li, and M. E. Davis Micropor. Mater. 2, pp. 17-26 (1993).

6. M. Ogawa, J. Am. Chem. Soc. 116, pp. $7941-7942$ (1994).

7. H. Yang, A. Kuperman, N. Coombs, S. Mamiche-Afara, and G. A. Ozin, Nature 379, pp. 703-705 (1996).

8. J. R. Bontha, A. Y. Kim, and J. Liu, Mater. Res. Soc. Symp. Proc., this volume.

9. D. H. Olson, G. D. Stucky, J. C. Vartuli, U.S. Patent No. 5364 797.(1994).

10. M. T. Anderson, J. E. Martin, J. G. Odinek, P. P. Newcomer, and J. P. Wilcoxon, submitted to Chemistry of Materials.

11. A. J. Ricco, G. C. Fry, and S. J. Martin, Langmuir 5, pp. 273-276 (1989).

12. M. T. Anderson, J. E. Martin; J. G. Odinek, P. P. Newcomer, and J. P. Wilcoxon, Mater. Res. Symp. Proc., this volume. 